\title{
Modelo SEM basado en valores organizacionales y capital intelectual: un estudio realizado en entidades del sistema financiero peruano
}

\section{SEM model based on organizational values and intellectual capital: a study conducted in entities of the peruvian financial system}

\begin{abstract}
Luis Alberto Geraldo Campos es docente de la Universidad Peruana Unión (Perú), (luis.geraldo@upeu.edu.pe)
(https://orcid.org/0000-0002-8366-689X)

Dr. Juan Jesús Soria Quijaite es docente e investigador de la Universidad Peruana Unión (Perú), (jesussoria@upeu.edu.pe), (https://orcid.org/0000-0002-4415-8622)

Dr. Pedro Leonardo Tito Huamaní es docente principal e investigador de la Universidad Nacional Mayor de San Marcos (Perú), (ptitoh@unmsm.edu.pe), (https://orcid.org/0000-0002-2989-9203)
\end{abstract}

\begin{abstract}
Resumen
La investigación analiza el efecto de los valores organizacionales en las dimensiones de capital intelectual: capital humano, capital estructural y capital relacional. La importancia del estudio de los constructos radica en la generación de conocimiento, esto se ha convertido en un recurso fundamental para las empresas, llevándolas a preocuparse por lo intangible de la organización. Los valores organizacionales, como base fundamental de la organización, brinda soporte para el gremio empresarial, por lo tanto, existe la necesidad de abordar estos constructos. El estudio se realizó bajo la metodología de modelos de ecuaciones estructurales (SEM), donde se realizó un análisis exploratorio y confirmatorio, con una muestra de 207 funcionarios de 15 entidades financieras, y se basó en el instrumento del inventario de perfil en valores organizacionales, de igual modo, el instrumento de capital intelectual, tuvo la finalidad de determinar el efecto que tienen las variables endógenas sobre las exógenas. Los resultados muestran que los valores organizacionales tienen relación causal significativa con el capital intelectual, y los constructos; capital humano $(\mathrm{r}=0.90)$, relacional $(\mathrm{r}=0.63)$ y estructural $(\mathrm{r}=0.89)$ con un error cuadrático medio de aproximación (RMSEA) de 0.08 y una discrepancia mínima por grado de libertad (CMIN/df) de 2.398 que hace relevante el modelo confirmatorio. Finalmente, se encontró una relación causal significativa y positiva entre los ocho valores organizacionales, los mismos que están influenciados de manera positiva en el capital humano, estructural y relacional.
\end{abstract}

\begin{abstract}
The research analyzes the effect of organizational values on the dimensions of intellectual capital: human capital, structural capital and relational capital. The importance of the study of radical constructs in the generation of knowledge, this has become a fundamental resource for companies, leading them to worry about the intangible nature of the organization. Organizational values, as the fundamental basis of the organization, providing support for the business union, therefore, there is a need to address these constructs. The study was carried out under the methodology of structural equation models (SEM), where an exploratory and confirmatory analysis was performed, with a sample of 207 organizations from 15 financial entities, and was based on the instrument of the profile inventory in organizational values, Similarly, the intellectual capital instrument was determined to determine the effect that endogenous variables have on exogenous ones. The results that show organizational values have a significant causal relationship with intellectual capital, and constructs; human capital ( $r=0.90)$, relational ( $r=0.63)$ and structural $(r=0.89)$ with a mean square error of approximation (RMSEA) of 0.08 and a minimum discrepancy by degree of freedom (CMIN/df) of 2,398 which makes relevant the confirmatory model. Finally, a significant and positive causal relationship was found among the eight organizational values, which are positively influenced in human, structural and relational capital.
\end{abstract}

\section{Palabras clave I keywords}

Valores organizacionales, capital intelectual, capital humano, capital estructural, capital relacional, Ecuaciones estructurales, Modelo SEM, sistema financiero.

Organizational values, intellectual capital, human capital, structural capital, relational capital, Structural equations, SEM Model, financial system.

Cómo citar: Geraldo Campos, L. A., Soria Quijaite, J. J., y Tito Huamaní, P. L. (2020). Modelo SEM basado en valores organizacionales y capital intelectual: un estudio realizado en entidades del Sistema Financiero Peruano. Retos Revista de Ciencias de la Administración y Economía, 10(19), 5-28. https://doi.org/10.17163/ret.n19.2020.01 


\section{Introducción}

A través de los años, las intensas transformaciones disruptivas, impulsadas por las exigencias de la competitividad del mercado, la información y la globalización económica, han presentado grandes desafíos en la gestión de las organizaciones, emergiendo nuevas formas de diagnóstico en la comprensión del comportamiento empresarial; frente a esto, han surgido nuevos conceptos como es el capital intelectual (CI), el cual, brinda importantes aportes para la mejora continua, con el propósito de obtener ventaja competitiva sostenible e innovadora, a través de las tres dimensiones de capital: capital humano, capital estructural y capital relacional (Demuner et al., 2017; Morales, 2017; Oro et al., 2017).

La falta de conocimiento en las diferentes empresas respecto a la aplicación del capital intelectual, a través de los años, tuvo carencias marcadas en Sudamérica. Los países desarrollados en el mundo han dado mayor importancia al estudio científico de la gestión del conocimiento y capital intelectual, creándose la necesidad de ser más competitivas frente a un mercado de constante cambio, dado que, por la competitividad, innovación constante a través de la gestión del capital intelectual, ha servido de base para el desarrollo de las organizaciones y el logro del éxito empresarial (Monagas, 2012; Osorio, 2003; Pérez \& Coutín, 2005; Velásquez, 2015).

Bajo el constante cambio y la preocupación por el posicionamiento en el mercado, las organizaciones hoy en día conservan al detalle la parte intangible de la empresa, el cual, es parte de su plan estratégico en donde interviene el capital intelectual y los valores organizacionales, este último, es el soporte de la organización conjuntamente con las políticas, la visión y misión de la empresa (Estivalete \& Andrade, 2012; Oliveira \& Tamayo, 2004). Es importante abordar estos constructos, porque las empresas financieras no dan énfasis al aporte que brindan los valores organizacionales. Asimismo, Velásquez (2007) menciona que si las organizaciones no consideran los valores organizacionales, tendrán un efecto negativo en el logro de los objetivos, como la presencia de conflictos, problemas con la adaptabilidad de los colaboradores, dificultades al momento de implantar una estrategia, dificultades en implantar un programa de mejoramiento, esto implica una baja productividad y calidad, entre otros problemas que impedirán alcanzar el éxito de la empresa.

Las empresas no se complicarían menciona, Camps (2015) cuando los valores deberían ser orientados a través de la conducta humana; sin embargo, con las organizaciones sucede lo mismo, debido a que no se enfatiza la práctica de valores. En contraste a lo señalado por Camps, Siles (2013) encontró una preferencia de los empresarios, en buscar colaboradores que fomenten y practiquen los valores éticos en el desarrollo de las actividades.

La preferencia de las empresas por los colaboradores con valores éticos se incrementa cada vez más, debido a que la práctica de valores influye en el comportamiento de los miembros y orientan al buen desempeño de la empresa, además de actuar como elementos integradores y con conocimientos de estrategias para el logro de objetivos (Alcover, Martínez, Rodríguez \& Domínguez, 2004; Oliveira \& Tamayo, 2004). Estos perfiles son considerados por los autores como un puente entre las características 
formales de la organización y el comportamiento individual, dado que se construye a través de las percepciones y creencias de los miembros de una organización.

Las dificultades de las organizaciones inteligentes son ocasionadas por falta de modelos de base para la orientación en los procesos y alineamientos del capital estructural, desarrollo del capital humano, capital relacional, estos nos llevan al crecimiento y cumplimiento de la visión de la organización. La investigación se realizó con una metodología correlacional múltiple con ecuaciones estructurales basadas en teorías (SEM) (Alaminos et al., 2015; Brown, 2015; Byrne, 2010; Catena et al., 2003; Hayes, 2013; Manzano \& Zamora, 2009; Raykov \& Marcoulides, 2006), con una muestra de 207 funcionarios de 15 entidades financieras, con el objetivo de determinar el efecto de los valores organizacionales en el capital intelectual. Estos constructos, engloban el grado de vínculo de los trabajadores y la organización. Hoy en día las grandes instituciones del sector financiero buscan ser más competitivas en todos los aspectos. El interés de esta investigación deriva del hecho de poder determinar el aporte que brindan a la organización. Es por eso, el estudio responde a las hipótesis que se planteó en base a los valores organizacionales (Oliveira \& Tamayo, 2004) y las tres dimensiones de capital intelectual (Martín et al., 2009), capital humano, capital estructural y capital relacional, desde una perspectiva intangible.

\subsection{Valores organizacionales}

Los valores organizacionales (VO) han predominado siempre en las empresas debido a la forma de cómo estos pueden orientar el comportamiento del colaborador (Demo et al., 2017), considerándose así como las convicciones y actitudes que enmarcan en la vida de los que integran una empresa (Hassan, 2007). Estos valores organizacionales se derivan de algunos supuestos básicos de naturaleza humana, los cuales, trabajan como el núcleo de las organizaciones, permitiendo así, provocar una marcada tendencia de dirección, integridad y autodisciplina en las personas (Chiavenato, 2009).

Los valores organizacionales forman parte del plan estratégico, donde se deben plantear claramente y luego darse a conocer a los colaboradores, con la finalidad que estén siempre presentes en el desenvolvimiento de las actividades como en los mecanismos, procesos, comportamientos y hasta en la estructura, permite alinear las estrategias en el cumplimiento de las metas y objetivos (Jaakson, 2010; Schein, 2004). Asimismo, los valores organizacionales ejercen un papel importante al atender a las necesidades de los individuos como también de atender los objetivos organizacionales (Tamayo, 2007), para esto, es de vital importancia que las empresas tenga reglado, internalizado y compartido los valores fundamentales para alcanzar las principales metas (Velásquez, 2007).

En la actualidad, las empresas están en constante cambio, bajo una compleja cultura influida por las creencias, estilos de vida y la forma de como las personas piensan y perciben al momento de relacionarse (Revilla, 2013). Estos elementos son básicamente agentes de cambio que impactan en la organización, es por ello que Oliveira y Tamayo (2004) agrupan los valores organizacionales en cuatro aspectos. 
Tabla 1. Aspectos que agrupan los valores organizacionales

\begin{tabular}{|l|l|}
\hline \multicolumn{1}{|c|}{ Tipos de aspectos } & \multicolumn{1}{c|}{ Descripción } \\
\hline Aspectos cognitivos & $\begin{array}{l}\text { Son las creencias de los colaboradores dentro de una organiza- } \\
\text { ción y la forma de cómo estos conciben la realidad y la respues- } \\
\text { ta de los problemas de la empresa. }\end{array}$ \\
\hline Aspectos motivacionales & $\begin{array}{l}\text { Estos aspectos muestran los intereses y deseos de los colabora- } \\
\text { dores en un determinado equipo de trabajo. }\end{array}$ \\
\hline Función de los valores & $\begin{array}{l}\text { La función de los valores, permite orientar la vida de las perso- } \\
\text { nas y delimitando la forma de pensar y actuar. }\end{array}$ \\
\hline Jerarquización de valores & $\begin{array}{l}\text { En cuanto a la jerarquía, este implica la preferencia y distinción } \\
\text { entre lo importante y lo secundario. }\end{array}$ \\
\hline
\end{tabular}

Fuente: Oliveira y Tamayo (2004)

Sin embargo, Oliveira y Tamayo (2004) para una mejor precisión de los valores propuestos por la teoría de Schwartz (1992), realizó un estudio con el objetivo de determinar un inventario de perfiles sobre los valores organizacionales, los cuales se describen en la tabla 2.

Tabla 2. Inventario de perfiles de valores organizacionales (IPVO)

\begin{tabular}{|l|l|}
\hline Valores organizacionales & \multicolumn{1}{c|}{ Criterios } \\
\hline Realización (RLZ) & $\begin{array}{l}\text { Basado en los elementos sobre competencias de los cola- } \\
\text { boradores que son determinantes para alcanzar el éxito. }\end{array}$ \\
\hline Conformidad (COF) & $\begin{array}{l}\text { Basado en las reglas y normas de comportamiento de los } \\
\text { colaboradores en función a otras organizaciones. }\end{array}$ \\
\hline Dominio (DOM) & $\begin{array}{l}\text { Basado en el poder, la obtención del estatus, el control so- } \\
\text { bre las personas y los recursos del mercado. }\end{array}$ \\
\hline Bienestar del empleado (BIEN) & $\begin{array}{l}\text { Basado en propiciar satisfacción a sus colaboradores, so- } \\
\text { bre todo su calidad de vida. }\end{array}$ \\
\hline Tradición (TRAD) & $\begin{array}{l}\text { Basado en la búsqueda de la preservación de las costum- } \\
\text { bres y prácticas de la organización. }\end{array}$ \\
\hline Prestigio organizacional (PRET) & $\begin{array}{l}\text { Basado en la búsqueda de prestigio e influencia en la so- } \\
\text { ciedad debido a la calidad de sus productos. }\end{array}$ \\
\hline Autonomía (AUT) & $\begin{array}{l}\text { Basado en el perfeccionamiento constante a través de la } \\
\text { valorización de la creatividad, experiencia, competencias } \\
\text { y curiosidad. }\end{array}$ \\
\hline $\begin{array}{l}\text { Preocupación por la colectivi- } \\
\text { dad (PPC) }\end{array}$ & $\begin{array}{l}\text { Basado en las relaciones que mantiene la empresa con los } \\
\text { individuos y con la comunidad. }\end{array}$ \\
\hline
\end{tabular}

Fuente: Oliveira y Tamayo (2004) 
Estos valores abordados son muy importantes como otros elementos que conforman la empresa, debido a que estarán siempre reflejados al momento de operar en el mercado. Además, las empresas del sector financiero deben implementar o ajustar sus valores organizacionales de acuerdo a los cambios surgidos durante el tiempo, permitiendo alinear a los colaboradores en un eficaz desempeño (Villalobos, 2014), esto le facilitará a la empresa tener funcionarios capaces de ampliar mejor sus habilidades e incrementar sus contribuciones para la entidad.

Los valores organizacionales son una pieza clave, y que según Ramírez, Sánchez, y Quintero (2005) deberán ser comunicados, divulgados y adoptados como valores individuales, desde allí valgan de base para las creencias, actitudes, opiniones, "percepciones" y conductas de la organización, produciendo una identidad y una sólida imagen corporativa en el mercado competente (Estivalete \& Andrade, 2012). A comparación con (Ramírez et al., 2005; Velásquez et al., 2012) alega que los valores organizacionales vienen a ser principios básicos para el cumplimiento de los objetivos, ya que, al ser asumidos por la empresa vendrán a tener un efecto en el comportamientos de los colaboradores, generando un soporte para la gerencia, promoviendo condiciones y pautas para el éxito de la empresa. Para Romero \& Izarra (2014), no solo son la base o principios, sino que también son la plataforma para la identidad y entendimiento de sus funcionarios, a su vez, los valores compartidos constituyen la raíz de la organización y generan ayuda para las personas y empresas que los ponen en práctica; además, estos deben estar presentes en cada empresa y deben ser comunicados durante las primeras etapas de incorporación del personal, no olvidando adiestrar constantemente al personal antiguo, empezando por los líderes de la empresa, debido a que estos son el ejemplo para sus colaboradores (Hernández et al., 2015; Javier \& Quintana, 2017; Ramírez et al., 2005; Yarce, 2000).

Finalmente, queda evidenciado del gran soporte y aporte que brindan los valores organizacionales en beneficio de la organización. El aprovechamiento está en comunicarlos y promoverlos de manera holística, teniendo como resultado un impacto positivo en el ámbito interno y externo, es decir, generando en los colaboradores un espíritu de compromiso y de diferenciación con los valores practicados en los colaboradores de otras empresas.

\subsection{Capital intelectual y sus dimensiones}

El capital intelectual (CI), es un concepto que ha sido utilizado desde hace varias décadas permitiéndoles a las empresas como investigadores profundizar mejor el tema (Sánchez et al., 2007), desde entonces, se han abordado diversas definiciones (Villegas et al., 2017), por lo que es necesario comprender el concepto y la importancia de este constructo.

Algunos investigadores trataron de definir el constructo, como es el caso de (Sánchez et al., 2007) donde indica que el capital intelectual es el "conocimiento y se convertirá de beneficio en el futuro y está formada por las ideas, los inventos, las tecnologías, los programas informáticos, los diseños y los procesos” (p.3), llegando hacer todo aquello de naturaleza intangible que cuenta la empresa (Rimbau-Gilabert \& Myrthianos, 2014); y está conformado por las habilidades y talentos, aplicaciones, patentes, los proveedores, la información del cliente y toda la experiencia que se 
puede construir, reconocer valorar y definir (Sánchez, 2012; Vidal, 2017), además, a estos elementos se incluyen, la capacidad para aprender y adaptarse, las marcas, los nombres de los productos y la capacidad de innovación y desarrollo, concluye (Sánchez et al., 2007). Es decir, el capital intangible es aquel capital que involucra elementos de carácter internos como aquellos que le permiten vincularse con lo externo de la empresa, es por eso, se divide en tres dimensiones, capital humano, capital estructural y capital relacional.

El capital intelectual ha evolucionado desde la década de los noventa, a través de diversos modelos propuestos, partiendo desde las dimensiones y los elementos que lo conforman, ver tabla 3.

\section{Tabla 3. Tipología del capital intelectual}

\begin{tabular}{|c|c|c|c|}
\hline Modelos/Autores & $\begin{array}{l}\text { Capital } \\
\text { Humano }\end{array}$ & $\begin{array}{c}\text { Capital } \\
\text { estructural }\end{array}$ & Capital Relacional \\
\hline $\begin{array}{l}\text { Cuadro de Mando } \\
\text { Integral (Kaplan y } \\
\text { Norton, 1993) }\end{array}$ & $\begin{array}{l}\text { Perspectiva de } \\
\text { Aprendizaje y } \\
\text { Crecimiento }\end{array}$ & $\begin{array}{l}\text { Perspectiva de Proce- } \\
\text { sos Internos }\end{array}$ & $\begin{array}{l}\text { Perspectiva de } \\
\text { clientes }\end{array}$ \\
\hline $\begin{array}{l}\text { Navegador de Skan- } \\
\text { dia (Edvisson, 1997) }\end{array}$ & $\begin{array}{l}\text { Enfoque } \mathrm{Hu}- \\
\text { mano }\end{array}$ & Enfoque de Procesos & Enfoque de Cliente \\
\hline $\begin{array}{l}\text { Monitor de los Activos } \\
\text { Intangibles (Sveiby, } \\
\text { 1997) }\end{array}$ & Competencias & Estructura Interna & Estructura Externa \\
\hline $\begin{array}{l}\text { Intelect (Euroforum, } \\
\text { 1998) }\end{array}$ & Capital Humano & Capital Estructural & Capital Relacional \\
\hline Intellectus (CIC, 2003) & Capital Humano & $\begin{array}{l}\text { Capital Tecnológico } \\
\text { Capital Organizativo }\end{array}$ & $\begin{array}{l}\text { Capital de negocio } \\
\text { Capital social }\end{array}$ \\
\hline $\begin{array}{l}\text { Chen, Zhu, y Yuan, } \\
2004\end{array}$ & Capital Humano & $\begin{array}{l}\text { Capital Innovación } \\
\text { Capital Estructural }\end{array}$ & Capital Cliente \\
\hline $\begin{array}{l}\text { Bueno, Salvador y } \\
\text { Rodríguez, } 2004\end{array}$ & Capital Humano & $\begin{array}{l}\text { Capital Tecnológico } \\
\text { Capital Organizativo }\end{array}$ & $\begin{array}{l}\text { Capital de negocio } \\
\text { Capital social }\end{array}$ \\
\hline $\begin{array}{l}\text { Guthrie, Petty, } \\
\text { Yongvanich, } 2004\end{array}$ & Capital Humano & Capital Interno & Capital Externo \\
\hline Ordóñez Pablo, 2004 & Capital Humano & $\begin{array}{l}\text { Capital Estructural: } \\
\text { Capital Tecnológico y } \\
\text { Capital Organizativo. }\end{array}$ & Capital Relacional \\
\hline Joia, 2004 & Capital Humano & $\begin{array}{l}\text { Capital Estructural: } \\
\text { Capital Interno, Capi- } \\
\text { tal Externo y Capital } \\
\text { Innovación }\end{array}$ & \\
\hline
\end{tabular}

Fuente: Yangali y Quiroz (2018, p. 44). 
De acuerdo a los modelos propuestos, los autores agrupan intangiblemente en tres dimensiones: capital humano, capital estructural y capital relacional que aportan al capital intelectual (Brooking, 1996; Chahal \& Bakshi, 2016; Edvinsson \& Sullivan, 1996; Euroforum, 1998), estos se pueden observar en la figura1.

\section{Figura 1. Dimensiones del capital intelectual}

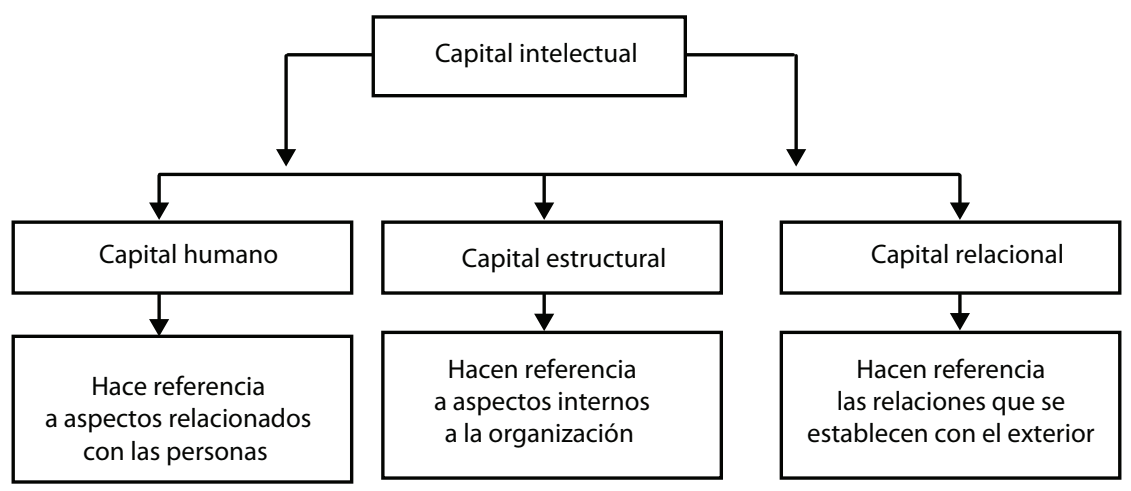

Fuente: Elaboración propia.

Estas dimensiones, permiten a los colaboradores emplear al máximo su capacidad de aprender y de innovar en la empresa, sobresaliendo el capital humano, cuyo elemento es el que predomina ante los otros dos capitales intangibles, los cuales deben ser aprovechados en su totalidad, a través de la mano de obra que posee la empresa (Rimbau-Gilabert \& Myrthianos, 2014).

\subsubsection{Capital humano}

El capital humano $(\mathrm{CH})$, es la dimensión principal del capital intelectual. Edvinsson y Malone (1998) resaltan que está constituido por las capacidades, actitudes, destrezas y conocimientos que cada miembro de la empresa aporta, y que estos no pueden ser propiedad de la empresa. Martín et al. (2009) indican que este elemento, es el conjunto de conocimientos básicos y propios de las personas que ayudan a mejorar la actividad de la empresa, teniendo como particularidad propia la imposibilidad de separar los activos intangibles de las personas que los desarrollan.

Este tipo de capital, suele centrarse en el carácter de los colaboradores a través de sus conocimientos, habilidades y esfuerzos, incrementando su productividad (Sen, 1998), este a su vez, suele llegar a la empresa de forma individual, al momento de reclutar los nuevos talentos para la organización (Fernández, Montes, \& Vázquez, 2010), algunos colaboradores son reubicados o ascendidos por el hecho que han invertido en gestión del conocimiento a través de experiencia y capacitación de manera efectiva y activa, permitiéndoles ampliar sus reservas de capital y tener mejores oportunidades, por ende, mayor posibilidad de lograr mayores retornos en el futuro para la empresa (Araújo, 2015; Marchante \& Ortega, 2010). 
Sin embargo, para que la empresa incremente su rentabilidad, esta deberá aprovechar al máximo el capital humano, ya que los colaboradores concentran una gran capacidad innovadora (Pizarro et al., 2011) no poner mayor énfasis en el valor que aporta, porque se corre el riesgo que ese capital humano sea transferible llevándose fácilmente el Know-how hacia otras empresas. Entonces, para que este capital humano no sea transferible, se sugiere que la empresa invierta en su talento para incrementar la productividad, la rentabilidad y obtener mayor innovación del colaborador (Kido \& Kido, 2015; Martín et al., 2009).

En la búsqueda de estudios recientes que relacionen el capital humano con los valores organizacionales, solo se encontró pequeñas grietas de contenido que vinculan estos constructos. Por su parte, Williams (2002) manifiesta que los valores organizacionales ( ) sirven como un mecanismo de vinculación entre los colaboradores, es decir, con el capital humano. Para Fitzgerald y Desjardins (2004) indican que si los colaboradores tienen valores organizativos bien definidos y comunicados, estarán más involucrados y más participativos en la toma de decisiones de la organización.

Entonces, bajo la problemática a estudiar estos constructos de manera conjunta y de corroborar con la escasa teoría, se plantea el modelo teórico de capital humano y valores organizacionales en base a las hipótesis:

H0ch: No existe un efecto significativo entre valores organizacionales y el capital humano H1ch: Existe un efecto significativo entre valores organizacionales y el capital humano

\subsubsection{Capital estructural}

El capital estructural (CE), es aquella dimensión que está basado en sistemas, procedimientos, bases de datos y constituye la forma más explícita de capital intelectual (Rimbau-Gilabert \& Myrthianos, 2014); es decir, es aquel conocimiento que la empresa adquiere y del cual se encarga de analizarlo, sistematizarlo e internalizarlo (Euroforum, 1998), generando para sí ventaja competitiva diferenciadora en la empresa, cuyo capital reside y queda impregnado dentro de la organización (Fernández-Jardón \& Martos, 2016; Ibarra-Cisneros \& Hernández-Perlines, 2019)

Entonces, al quedar este capital dentro de la organización, va influir en otros activos intangibles, permitiendo un flujo de conocimiento y perfeccionando la eficacia al construir de manera adecuada los diversos trabajos de la organización, por tanto, dependerá del tamaño y la antigüedad para que este capital sea más rentable para la empresa (Edvinsson \& Malone, 2004; Herrera \& Macagnan, 2015), además, será un recurso esencial para hacer frente a la competencia (Fernández-Jardón \& Martos, 2016).

Se han venido postulando diversos modelos de capital intelectual, lo que ha permitido que algunos de los investigadores en esta línea, lleguen a dividir al capital estructural en dos tipos de capital. 
Tabla 4. Tipos de capital estructural

\begin{tabular}{|l|l|}
\hline \multicolumn{1}{|c|}{ Tipos } & \multicolumn{1}{c|}{ Hace referencia } \\
\hline Capital organizativo & $\begin{array}{l}\text { Al diseño estructural, al proceso coordinado, como a las rutinas or- } \\
\text { ganizativas, a la cultura y comportamiento mismos del equipo de } \\
\text { trabajo, la planificación y control de las actividades. }\end{array}$ \\
\hline Capital tecnológico & $\begin{array}{l}\text { Las patentes, el know-how, la propiedad industrial que posee, como } \\
\text { también el secreto industrial y la experiencia técnica que la empresa } \\
\text { ha adquirido. }\end{array}$ \\
\hline
\end{tabular}

Fuente: Navas y Ortiz (2002)

Bajo estos dos capitales está enfocado el capital estructural, siendo que hoy en día, este capital se ve reflejado en las empresas a pesar de que muchas de estas solo se encuentran operando en el internet (Eyzaguirre, 2017; Mercado-Salgado, 2016; Vidal, 2017). Esto permite, la creación de nuevas estrategias de capital organizativo y tecnológico, motivando a una mejora continua por parte del sector empresarial.

Sin embargo, al igual que el capital humano, existen escasos estudios que vinculen al capital estructural con los valores organizacionales. El capital humano es precisamente un intermediario que permite vincular el capital estructural con los valores organizacionales, siendo los colaboradores quienes intervienen sobre los procesos y tareas que demandan interacción social (Arciniega, Woehr \& Poling, 2008); en tal sentido, los valores organizacionales, se ven reflejados en los procesos, mecanismos, comportamientos y estructuras de la organización, con el propósito de alcanzar los objetivos y metas planificadas (Jaakson, 2010; Navas \& Ortiz, 2002; Rimbau-Gilabert \& Myrthianos, 2014; Sánchez et al., 2007; Schein, 2004). Entonces, los VO con el CE, también se verán vinculadas con los sistemas, procedimientos y bases de datos (Rimbau-Gilabert \& Myrthianos, 2014). Partiendo de esta premisa existe un vínculo con el capital organizativo y el capital tecnológico (Navas \& Ortiz, 2002), con la finalidad de poder complementarse al momento de efectuar cualquier actividad durante el ejercicio económico de la organización.

Entonces, bajo la problemática de aclarar mejor los postulados teóricos, se establece el modelo teórico de capital estructural y los valores organizacionales en base a las siguientes hipótesis:

H0ce: No existe un efecto significativo entre valores organizacionales y capital estructural H1ce: Existe un efecto significativo entre valores organizacionales y capital estructural

\subsubsection{Capital relacional}

Uno de los tres capitales es el capital relacional (CR), el cual, consiste en la relación que tiene la empresa con los proveedores, los clientes y agentes externos que han podido establecer contacto con la empresa durante el tiempo. Por ser transcendental en la toma de decisiones a través de herramientas tecnológicas, apoyado por el capital humano y capital estructural, este influye directamente con el capital relacional apoyado en la marca, la lealtad y las relaciones mismas con los proveedores (Kogut 
\& Zander, 1996; Martín et al., 2009; Martínez \& Cegarra, 2003; Navas \& Ortiz, 2002; Rimbau-Gilabert \& Myrthianos, 2014).

De la misma forma, influye en aquellos activos intangibles que obtiene la empresa cuando mantiene relaciones con agentes de su entorno como clientes, proveedores y aliados (Alzate Ortiz, \& Jaramillo Arenas, 2015), produciéndose un conocimiento superior que surge en base la coordinación y combinación de parte del conocimiento propio de cada uno de los agentes que intervienen en la relación. Además, ofrece información acerca de los intereses que muestran los agentes de su entorno los cuales resultan decisivos para detectar oportunidades tecnológicas o de mercado que guíen su proceso de desarrollo de nuevos conocimientos (Martín et al., 2009).

En base a los intereses de los agentes, Navas y Ortiz (2002) clasificaron en dos tipos de agentes, haciendo referencia a los agentes externos y los agentes internos, no dejando de lado al denominado capital de mercado, el cual, recoge las relaciones externas de la empresa, consideradas desde un punto de vista muy amplio. Cabe enfatizar, que las empresas siempre buscarán establecer nexos con empresas de otros sectores incluyendo las de la competencia. En esto el capital humano juega un papel importante, el cual, va ampliar la red de contactos con esas empresas y que le permitirá cumplir con sus propósitos estratégicos establecidos.

En la búsqueda de vincular el capital relacional con los valores organizacionales, Revilla (2013) indica que los valores organizacionales tienen un vínculo al momento relacionarse con las personas y esto se ve reflejado al momento de entrar en contacto con los clientes internos y externos (Navas \& Ortiz, 2002; Revilla, 2013; Santos et al., 2011), es decir, en la relación con los clientes, proveedores y aliados (Kogut \& Zander, 1996; Martín et al., 2009; Martínez \& Cegarra, 2003; Navas \& Ortiz, 2002; Ortiz \& Arenas, 2015; Rimbau-Gilabert \& Myrthianos, 2014).

Para corroborar estos postulados teóricos, esta investigación se centra en la problemática de establecer el modelo teórico de capital relacional y los valores organizacionales en base a las siguientes hipótesis:

H0cr: No existe un efecto significativo entre los valores organizacionales y el capital relacional

H1cr: Existe un efecto significativo entre valores organizacionales y capital relacional

\section{Materiales y método}

En este estudio, se analiza de manera global el efecto de los valores organizacionales sobre las dimensiones de capital intelectual. Para determinar el efecto de los valores organizacionales en capital humano, capital estructural y capital relacional, se utilizó un análisis multivariante con Modelos de Ecuaciones Estructurales (SEM) de acuerdo a (Alaminos et al., 2015; Brown, 2015; Byrne, 2010; Catena et al., 2003; Hayes, 2013; Manzano \& Zamora, 2009; Raykov \& Marcoulides, 2006; Saboya et al., 2019). Este tipo de análisis examina simultáneamente una serie de relaciones de dependencia con la finalidad de desarrollar perspectivas más sistemáticas y holísticas de los problemas tratados, además de originar una reflexión más profunda (Alaminos et 
al., 2015; Hair et al., 2007; Manzano \& Zamora, 2009). Se planteó el modelo teórico representado en la figura 2.

Figura 2. Secuencia gráfica de relaciones de los constructos

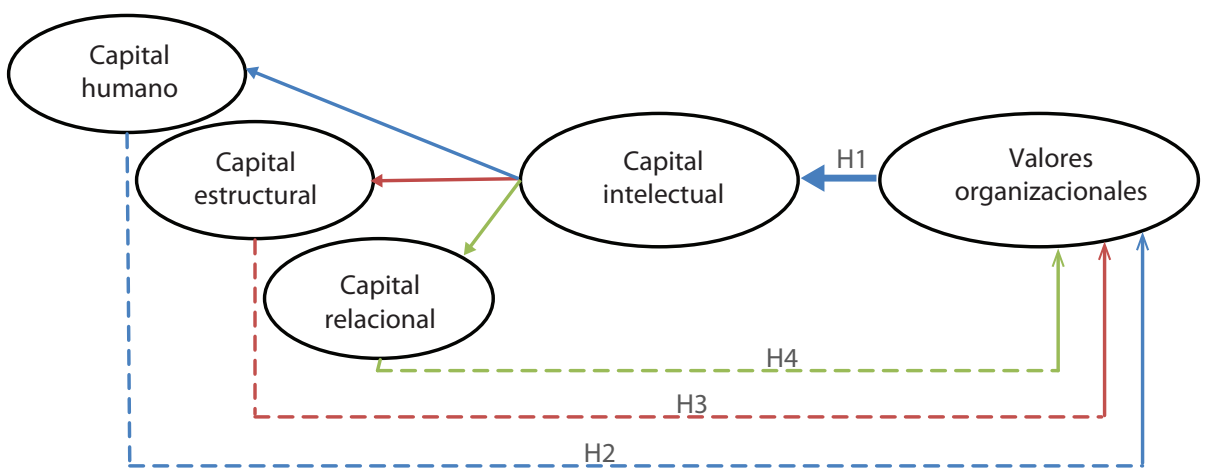

Fuente: Elaboración propia.

Para el análisis del Modelos (SEM) se planteó el Modelo de regresión estructural que permite la asociación entre variables latentes (Manzano \& Zamora, 2009)

Modelo estructural:

$$
\eta=\mathrm{B \eta}+\Gamma \mathrm{X}+\zeta(1)
$$

Modelo de medición:

$$
\begin{aligned}
& \mathrm{Y}=\Lambda_{\mathrm{X}} \eta+\varepsilon(2) \\
& \mathrm{X}=\Lambda_{\mathrm{Y}} \xi+\delta(3)
\end{aligned}
$$

La población de estudio consta de objetos o sujetos que tienen una cantidad o características (Ghozali, 2006). En la investigación la población de estudio fueron 15 entidades organizacionales, como se muestra en la tabla 5, que forman parte del sistema financiero peruano entre Bancos, Cooperativas, Financieras y Cajas Rurales y Municipales, el cual se aplicó el instrumento de valores organizacionales (Oliveira \& Tamayo, 2004) y capital intelectual (Martín et al., 2009) a 207 funcionarios de mandos altos, mandos medios y operativos con una vinculación no menor a 6 meses. Los instrumentos fueron administrados por los investigadores, presentándose 15 solicitudes a las organizaciones en estudio y previa autorización se ejecutó, con una duración de 05 meses, iniciando el 12 de enero al 04 de mayo del 2019. 
Tabla 5. Muestreo de las entidades financieras

\begin{tabular}{|c|c|c|}
\hline $\mathbf{N}^{\circ}$ & Entidad financiera & Encuestados \\
\hline 1 & Banco de Crédito del Perú & 16 \\
\hline 2 & Scotiabank & 13 \\
\hline 3 & Financiera Crediscotia & 20 \\
\hline 4 & Caja Maynas & 18 \\
\hline 5 & Mibanco & 20 \\
\hline 6 & Caja Trujillo & 16 \\
\hline 7 & Cooperativa San Martin de Porres & 18 \\
\hline 8 & Financiera Confianza & 13 \\
\hline 9 & Banco de la Nación & 1 \\
\hline 10 & Interbank & 15 \\
\hline 11 & BBVA Banco Continental & 10 \\
\hline 12 & Caja Metropolitana & 3 \\
\hline 13 & Caja Paita & 16 \\
\hline 14 & Caja Piura & 15 \\
\hline 15 & Cooperativa de Ahorro y Crédito la Progresiva & 13 \\
\hline \multicolumn{2}{|c|}{ Total de muestra } & 207 \\
\hline
\end{tabular}

Fuente: Elaboración propia.

Como lo sugiere Westland (2019) antes de las pruebas descriptivas y positivas, es importante analizar los datos, esto, por la naturaleza de las variables del presente estudio que son ordinales con respuestas de escala Likert. Westland señala que la selección ayudará en el aislamiento de las peculiaridades de los datos y permitirá que los datos se ajusten antes de un análisis multivariado adicional. Además, se considera lo sugerido por Tabachnick, Fidell, y Ullman (2007) de revisar previamente los datos, es decir, los supuestos para un previo análisis.

En el análisis de los datos se usó la herramienta complementaria del Statistical Product and Service Solutions (SPSS) denominada AMOS versión 25, con la finalidad de compilar el modelo teórico propuesto por los investigadores (ver figura 3), buscando la validación a las hipótesis teóricas planteadas. 
Figura 3. Modelo general de las hipótesis teóricas

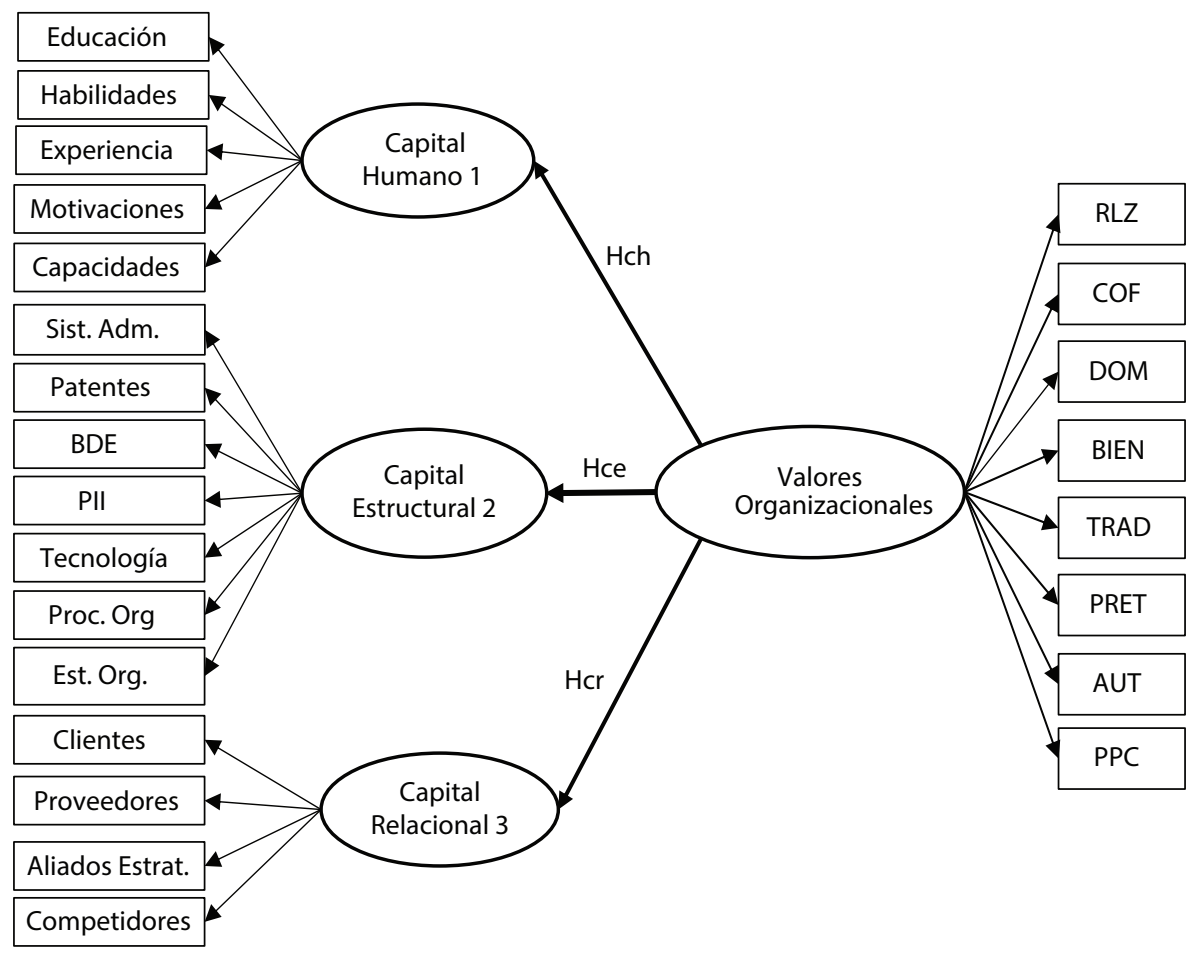

Fuente: Elaboración propia.

\section{Resultados}

\subsection{Caracterización y evaluación del modelo SEM}

En el modelo SEM de la figura 4 se identifican los valores estimados estandarizados del modelo general complejo, dando las condiciones suficientes para su estimación. De acuerdo a (Alaminos et al., 2015; Bollen, 1989; Browne \& Cudeck, 1992; Byrne, 2010; Catena et al., 2003; Manzano \& Zamora, 2009; Raykov \& Marcoulides, 2006; Schumacker \& Lomax, 2010) el modelo SEM utilizó medidas de ajuste global para validar el modelo teórico mostrado en la figura 8.

De acuerdo con la figura 4 donde las variables superficiales aportan una relación causal a las variables exógenas y estas influenciadas por las endógenas, se obtuvo una carga factorial estandarizada de $0.88,0.84$ y 0.64 respectivamente equivalente a los resultados de la tabla 6. Se determinó que el modelo SEM de Valores Organizacionales y los tres tipos de capital intelectual sin modificaciones como se muestra en la tabla 6 , se observa un valor Chi-square $=710.970$ y un nivel de probabilidad $\rho=0.000$ con 249 grados de libertad. 
Tabla 6. Medidas de ajuste global sin modificación

\begin{tabular}{|c|c|c|c|c|c|c|}
\hline $\begin{array}{c}\text { Medida de } \\
\text { ajuste }\end{array}$ & \multicolumn{1}{|c|}{$\mathbf{X}^{\mathbf{2}}$} & \multicolumn{1}{c}{ df } & \multicolumn{1}{c|}{ NP } & CMIN/df & \multicolumn{1}{c|}{ CFI } & RMSEA \\
\cline { 2 - 7 } & 710.970 & 249 & 0.000 & 2.855 & 0.815 & 0.103 \\
\hline
\end{tabular}

$X^{2}=$ Chi-square; $d f=$ Grados de libertad; NP = Nivel de Probabilidad CMIN/df = Chi-square/grados de libertad; GFI = índice de bondad de ajuste; RMSEA = Error Medio Cuadrático de Aproximación

Asimismo, las medidas de ajuste global tienen un CMIN/df igual a 2.855 que es relevante y un CFI igual a 0.815 que también es significativo a diferencia de un RMSEA igual a 0.103 que es significativo pero no está dentro de los parámetros permitidos según (Browne \& Cudeck, 1992; Schumacker \& Lomax, 2010).

\section{Figura 4. Modelo SEM valores organizacionales y los tipos de capital intelectual sin modificaciones}

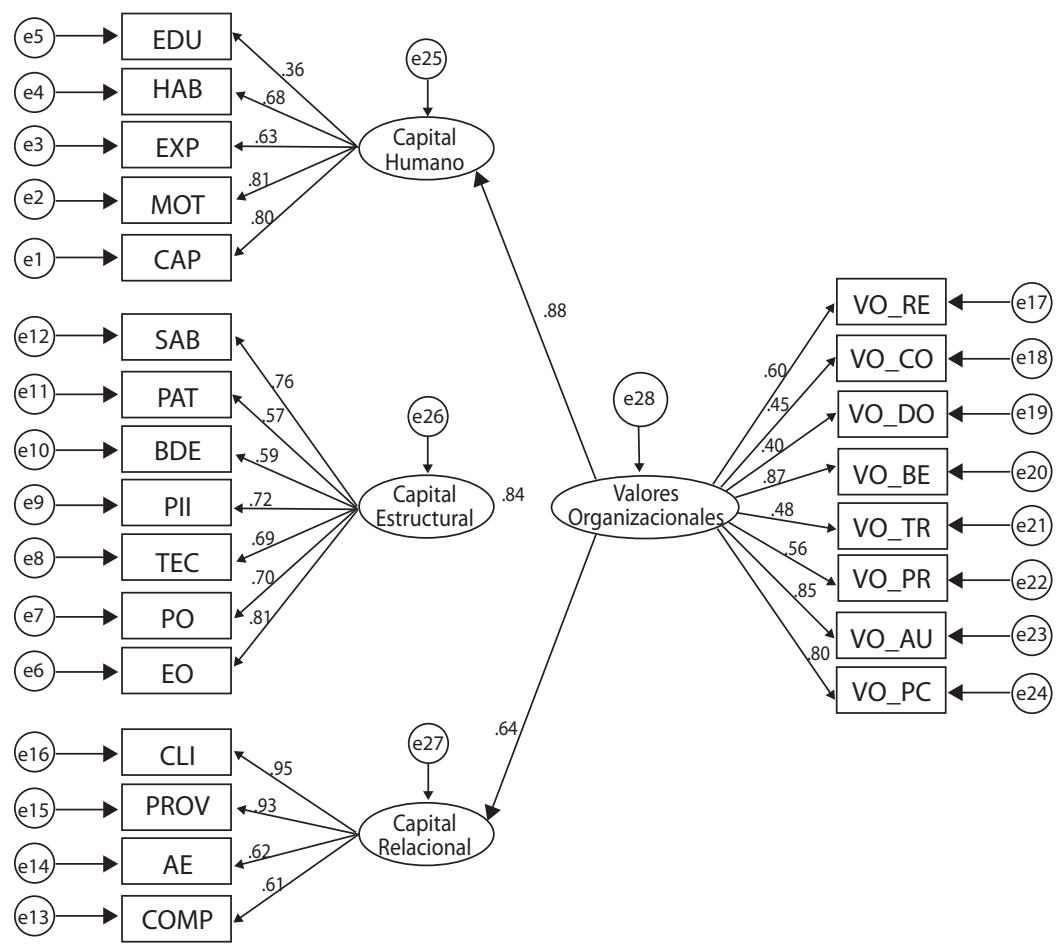

Fuente: Elaboración propia.

En la tabla 6 donde muestra un RMSEA que no está dentro de los parámetros permitidos, se hizo un reajuste de los errores del modelo SEM, obteniéndose el modelo de la figura 5. Se confirmó un modelo SEM de valores organizacionales y los tres tipos de capital intelectual (Capital Humano, Capital Estructural y Capital Relacional) tal 
como se muestra en la figura antes mencionada. Asimismo, se obtuvo un Chi-square $=$ 516.186 y un nivel de probabilidad $\rho=0.000$ que siendo menor al nivel de significancia $\alpha=0.05$ se valida el modelo SEM con $d f=199$ grados de libertad. Además, el modelo de ajuste global modificado (tabla 7) arrojó un CMIN/df igual a 2.594 que es relevante, un GFI igual a 0.805 , un CFI igual a 0.868 , un PCFI de 0.748 que son significativo respectivamente. Cabe enfatizar, el resultado del modelo de ajuste global modificado obtuvo un RMSEA igual a 0.079, estando dentro de los parámetros establecidos (el valor de .05 a .08 indica un ajuste perfecto) según (Browne \& Cudeck, 1992; Schumacker \& Lomax, 2010), se acepta el nuevo modelo SEM de la figura 5.

Tabla 7. Modelo de ajuste global Modificado

\begin{tabular}{|c|c|c|l|l|l|l|l|}
\hline $\mathbf{X}^{\mathbf{2}}$ & df & NP & CMIN/df & GFI & CFI & PCFI & RMSEA \\
\hline 570.688 & 238 & 0.000 & 2.398 & 0.798 & 0.867 & 0.748 & 0.08 \\
\hline
\end{tabular}

$X^{2}=$ Chi-square; $d f=$ Grados de libertad; NP = Nivel de Probabilidad; CMIN/df = Chi-square/grados de libertad; GFI = índice de bondad de ajuste; $\mathrm{CFI}=$ índice de ajuste comparativo; PCFI = Medidas Ajustadas de Parsimonia; RMSEA = Error Medio Cuadrático de Aproximación.

\section{Figura 5. Modelo SEM de los valores organizacionales} y los tres tipos de capital intelectual con modificaciones

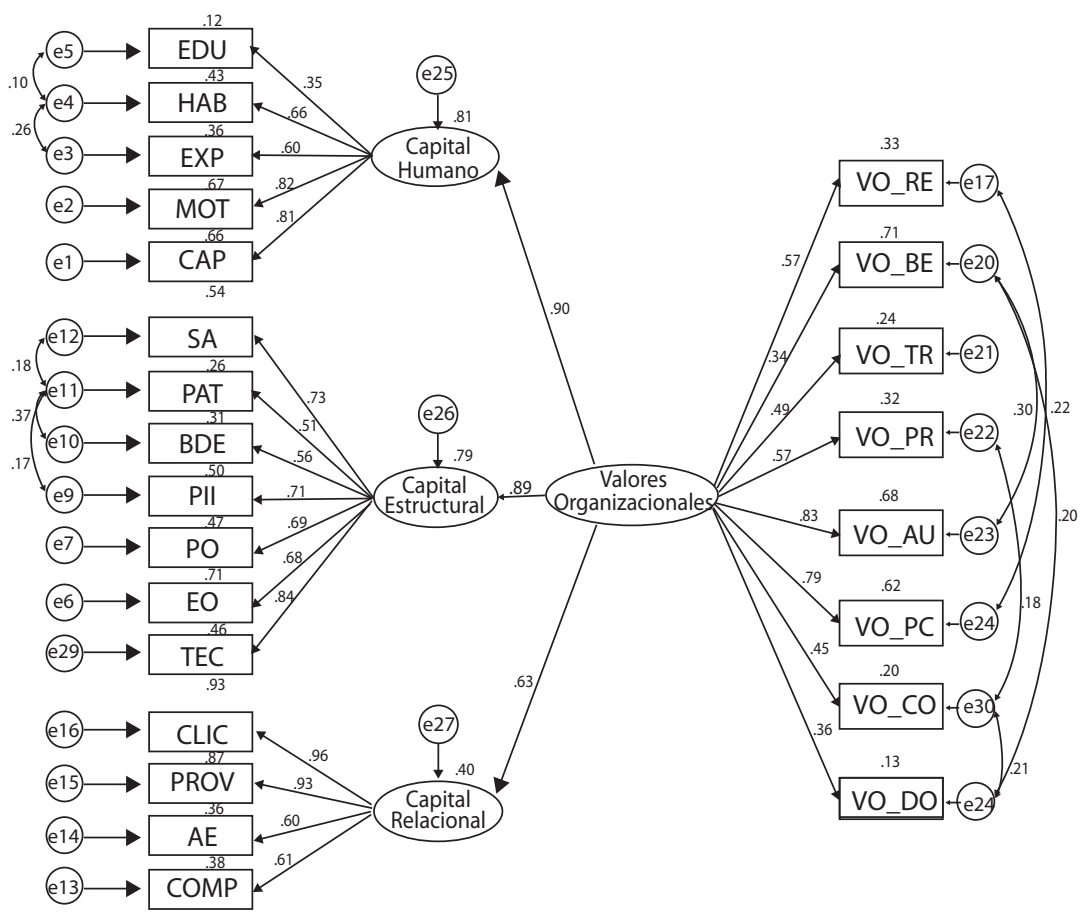

Fuente: Elaboración propia. 
En la figura 5 observamos que la variable endógena, capital humano tiene una relación causal con las variables superficiales, las capacidades (CAP) con un estimador estandarizado (relación causal) de 0.812, las motivaciones (MOT) con un estimador estandarizado de 0.816 , las habilidades (HAB) con un estimador estandarizado de 0.659 y las experiencias (EXP) con un valor estandarizado de 0.600 de los colaboradores en estudio. Asimismo, el indicador de educación (EDU) tiene un valor estandarizado de 0.346 y observando el p-valor se observa que este valor aporta una relación causal al capital humano, el cual se debe repotenciar con capacitación y otros indicadores relacionados a la educación.

La segunda variable endógena que es el capital estructural tiene una relación causal con sus indicadores más significativos como la estructura organizativa (EO) con una relación causal de 0.843 , el sistema administrativo (SA) con una relación causal de 0.733 , la propiedad intelectual e industrial (PII) con una relación causal 0.708 , el proceso organizativo (PO) con una correlación de 0.688 , la tecnología (TEC) con una relación causal de 0.676 , la base de datos estratégicos (BDE) con una correlación de 0.557, y finalmente las patentes (PAT) con una correlación causal de 0.507.

En la tercera variable endógena que es el capital relacional tiene una relación causal con sus indicadores más significativos como los clientes (CLI) con una correlación causal de 0.963 , los proveedores (PROV) con una relación causal de 0.933 , los competidores (COMP) con una relación causal de 0.613 y finalmente los aliados (AE) tienen una correlación causal de 0.598 .

Por otro lado, la variable exógena valores organizacionales muestra las cargas más relevantes de las variables superficiales exógenas, donde la concentración de categorización es el bienestar (VO_BE) con una correlación causal de 0.840, la autonomía (VO_AU) con una correlación causal de 0.825, la preocupación por la colectividad (VO_PC) con una correlación causal de 0.787, la realización (VO_RE) con una relación causal de 0.575 , el prestigio de la empresa (VO_PR) con una relación causal de 0.567 y el menos significativo la tradición (VO_TR) relación causal de 0.491 , la conformidad (VO_CO) con una relación causal de 0.446 y el dominio (VO_DO) con una relación causal de 0.358 , el cual no se ha dado mucho énfasis a estos valores respectivamente.

\subsection{Comprobación y validación de las hipótesis planteadas}

La figura 3 está formulada por supuestos (Hch, Hce y Hcr) con el modelo estructural teórico de ecuaciones estructurales SEM, la figura 4 confirma que existen correlaciones causales de influencia y predicción entre las variables exógenas y las endógenas en estudio, pero se tuvo modificaciones debido a que los índices de bondad de ajuste no estaban dentro de los parámetros establecidos según la teoría (Browne \& Cudeck, 1992; Catena et al., 2003; Schumacker \& Lomax, 2010), se tuvo un modelo SEM ajustado modificado en la figura 5.

Bajo el modelo estructural SEM confirmatorio modificado de la figura $5 \mathrm{y}$ los resultados de estimaciones estandarizadas como se muestra en la tabla 8, se aprecia que los estimadores son mayores a 0.5 a excepción de educación (EDU) con 0.346 con un p valor significativo. En tal sentido se corroboran la primera hipótesis teórica planteada rechazando la hipótesis nula H0ch y aceptando la alterna H1ch el cual confirma que existe un efecto significativo entre los valores organizaciones y el 
capital humano con un índice de efectividad de 0.902. La segunda hipótesis planteada en el modelo teórico nos confirma H1ce que existe un efecto significativo entre las variables exógenos valores organizacionales y la variable endógena capital estructural con un índice de efectividad de 0.887. Asimismo, la tercera hipótesis en el modelo de ecuaciones estructurales confirma que existe un efecto significativo entre los valores organizacionales y la variable capital relacional con un índice de efectividad de 0.633 .

Tabla 8. Pesos de regresión y estandarizados del Modelo predeterminado

\begin{tabular}{|c|c|c|c|c|c|c|c|}
\hline \multicolumn{3}{|c|}{ Relación causal } & Estimate & S.R.W. & S.E. & C.R. & $\mathbf{P}$ \\
\hline Capital_Humano & $<--$ & Valores_Organizacionales & .743 & .902 & .100 & 7.429 & $* * *$ \\
\hline Capital_Estructural & $<--$ & Valores_Organizacionales & 4.645 & .887 & .615 & 7.553 & $* * *$ \\
\hline Capital_Relacional & $<--$ & Valores_Organizacionales & .601 & .633 & .109 & 5.534 & $* * *$ \\
\hline CAP & $<--$ & Capital_Humano & 1.000 & .812 & & & \\
\hline MOT & $<--$ & Capital_Humano & 1.753 & .816 & .149 & 11.728 & $* * *$ \\
\hline НAB & $<--$ & Capital_Humano & 1.823 & .659 & .202 & 9.006 & $* * *$ \\
\hline EDU & $<--$ & Capital_Humano & .718 & .346 & .163 & 4.416 & $* * *$ \\
\hline EXP & $<--$ & Capital_Humano & 1.828 & .600 & .227 & 8.064 & $* * *$ \\
\hline EO & $<---$ & Capital_Estructural & 1.000 & .843 & & & \\
\hline $\mathrm{PO}$ & $<--$ & Capital_Estructural & .174 & .688 & .017 & 10.002 & $* * *$ \\
\hline PII & $<--$ & Capital_Estructural & .515 & .708 & .050 & 10.399 & $* * *$ \\
\hline $\mathrm{BDE}$ & $<--$ & Capital_Estructural & .087 & .557 & .011 & 7.680 & $* * *$ \\
\hline PAT & $<--$ & Capital_Estructural & .097 & .507 & .014 & 6.799 & $* * *$ \\
\hline SA & $<--$ & Capital_Estructural & .219 & .733 & .020 & 10.902 & $* * *$ \\
\hline TEC & $<--$ & Capital_Estructural & .110 & .676 & .011 & 9.785 & $* * *$ \\
\hline COMP & $<--$ & Capital_Relacional & 1.000 & .613 & & & \\
\hline $\mathrm{AE}$ & $<--$ & Capital_Relacional & 1.004 & .598 & .099 & 10.144 & $* * *$ \\
\hline PROV & $<--$ & Capital_Relacional & 1.357 & .933 & .142 & 9.555 & $* * *$ \\
\hline CLI & $<--$ & Capital_Relacional & 2.514 & .963 & .262 & 9.609 & $* * *$ \\
\hline VO_TR & $<--$ & Valores_Organizacionales & 1.598 & .491 & .287 & 5.563 & $* * *$ \\
\hline VO_PR & $<--$ & Valores_Organizacionales & 1.237 & .567 & .199 & 6.206 & $* * *$ \\
\hline VO_PC & $<--$ & Valores_Organizacionales & 4.482 & .787 & .520 & 8.614 & $* * *$ \\
\hline VO_AU & $<--$ & Valores_Organizacionales & 4.255 & .825 & .537 & 7.922 & $* * *$ \\
\hline VO_RE & $<--$ & Valores_Organizacionales & 1.000 & .575 & & & \\
\hline VO_BE & $<--$ & Valores_Organizacionales & 3.298 & .840 & .412 & 8.003 & $* * *$ \\
\hline VO_CO & $<--$ & Valores_Organizacionales & .855 & .446 & .166 & 5.137 & $* * *$ \\
\hline VO_DO & $<--$ & Valores_Organizacionales & .546 & .358 & .129 & 4.249 & $* * *$ \\
\hline
\end{tabular}

S.R.W. = Standardized Regression Weights; S.E.=Estimate Standardized 


\section{Conclusiones y discusión}

Los resultados del estudio revelan una relación causal significativa y positiva entre los ocho valores organizacionales (RLZ, COF, DOM, BIEN, TRAD, PRET, AUT y PPC), los mismos que están influenciando de manera positiva en el capital humano, capital estructural y capital relacional respectivamente.

En primera instancia la relación causal que tienen los valores organizacionales sobre el capital humano permiten enfatizar que existe buena práctica de los valores organizacionales por parte de los funcionarios, esto se debe a que los valores han sido compartidos (Alcover, Martínez, Rodríguez, \& Domínguez, 2004; Oliveira \& Tamayo, 2004), divulgados (Ramírez et al., 2005) e interiorizados (Velásquez, 2007). En tal sentido, el capital humano guarda un gran respeto por preservar la marca y practicar buenas costumbres (Oliveira \& Tamayo, 2004), esto se verán pronunciadas al momento de vincularse con la estructura misma de la entidad (CE2) (Jaakson, 2010; Schein, 2004), es decir, los sistemas administrativos, patentes, base de datos y propiedad intelectual de la empresa (Navas \& Ortiz, 2002; Rimbau-Gilabert \& Myrthianos, 2014), repercutiendo en el vínculo que sus miembros puedan tener con los agentes externos (CR3) (Kogut \& Zander, 1996; Martín et al., 2009; Martínez \& Cegarra, 2003; Navas \& Ortiz, 2002; Rimbau-Gilabert \& Myrthianos, 2014) respetando la buena práctica de valores (Hassan, 2007) como las políticas de cultura tradicional de la empresa.

El efecto que tuvo los valores organizacionales expuestos en el modelo SEM modificado frente al capital humano, la relación causal es mayor que el capital estructural y relacional, debido a que este capital humano es el que predomina ante los demás capitales (Rimbau-Gilabert \& Myrthianos, 2014) y se preocupa por prestar atención e interacción con los individuos en atender los objetivos organizacionales (Tamayo, 2007). Estas afirmaciones, confirma que el capital humano es influenciado por los valores de la organización, mientras se lleguen a divulgar (Velásquez, 2007), compartir y practicar por todos los miembros de la entidad (Alcover et al., 2004; Oliveira \& Tamayo, 2004).

El efecto de los constructos mostrados en la tabla 7 y el modelo SEM de la figura 10, permite enfatizar que los valores organizacionales están orientando mejor a la vida y el comportamiento de las funcionarios (Camps, 2015; Demo et al., 2017), delimitándose la forma de pensar y actuar (Oliveira \& Tamayo, 2004), reflejando en ellos las capacidades (Sánchez et al., 2007), actitudes, destrezas y conocimientos (Edvinsson \& Malone, 2004; Hassan, 2007) que cada funcionario de la entidad financiera posee y aporta para ésta. En ese sentido, al existir un vínculo entre los valores organizacionales y el capital humano, se evidencia que los funcionarios son valorados, y se desarrollan profesionalmente dentro de la entidad financiera, aportando con sus destrezas, capacidades, habilidades y conocimientos (Edvinsson \& Malone, 2004; León \& Mancheno, 2017; Sarur, 2013; Valencia, 2005), para el logro de objetivos y metas de la entidad financiera donde estos se vienen desempeñando.

En segunda instancia, los valores organizacionales influyen en el capital estructural, siendo el capital humano un vínculo de estos constructos, es decir, los colaboradores influidos por los valores organizacionales arraigados intervienen sobre los procesos y tareas que demandan interacción social (Arciniega et al., 2008); ade- 
más, los valores organizacionales conducidos por el capital humano, se ven reflejados en los procesos, mecanismos, comportamientos y estructuras de la organización, con la finalidad de lograr alcanzar los objetivos y metas planificadas (Jaakson, 2010; Navas \& Ortiz, 2002; Rimbau-Gilabert \& Myrthianos, 2014; Sánchez et al., 2007; Schein, 2004). Los resultados del modelo de SEM permite corroborar que los valores organizacionales se verán vinculadas con los sistemas, procedimientos y bases de datos (Rimbau-Gilabert \& Myrthianos, 2014), es decir, partiendo de esta premisa existe un vínculo con el capital organizativo y el capital tecnológico (Navas \& Ortiz, 2002), con la finalidad de poder complementarse al momento de efectuar cualquier actividad durante el ejercicio económico de la organización.

Como se verificó que los valores organizacionales tienen una relación causal en el capital relacional y este a su vez presentando valores altos en las variables superficiales, clientes, proveedores, aliados estratégicos y competidores, son de igual modo conducidos por el capital relacional y apoyándose en el capital estructural. Se verifica con los autores (Kogut \& Zander, 1996; Martín et al., 2009; Martínez \& Cegarra, 2003; Navas \& Ortiz, 2002; Rimbau-Gilabert \& Myrthianos, 2014) que los valores organizacionales mencionados influyen en relación a la marca, la lealtad, y las relaciones mismas con los proveedores, y de acuerdo a (Ortiz \& Arenas, 2015) influyen directamente con los clientes, proveedores y aliados al momento que los agentes se relacionen con su entorno de la organización.

Los resultados permiten señalar que el capital relacional movido por el capital humano y los valores que lleva consigo, juega un papel importante al momento de relacionarse con agentes externos e internos; es decir, con los clientes, proveedores, aliados estratégicos e incluso con los competidores, ya que permitió ampliar la red de contactos de la empresa, por consiguiente, cumplir sus propósitos estratégicos establecidos. De acuerdo a los resultados expuestos en el modelo SEM se corrobora con (Navas \& Ortiz, 2002; Revilla, 2013; Santos et al., 2011) donde los valores organizacionales serán un efecto significativo en el capital relacional cuando estos se vean reflejados al momento de relacionarse con agentes externos e internos de la organización, por consiguiente.

Este estudio permite determinar que los valores organizacionales son practicados, divulgados e interiorizados por los colaboradores. Son el soporte para la administración que promueven condiciones y pautas para alcanzar el éxito de la financiera. Además, permiten afirmar que los valores organizacionales son la base fundamental de la organización. Los valores toman sentido en la organización cuando son comunicados, difundidos y promocionados lo suficientemente (Ramírez et al., 2005). Al mantener los valores organizacionales una relación causal positiva entre los dos constructos, se afirma que las financieras aprovechan al máximo el capital humano a través de la creación de conocimiento e innovación, plasmadas en nuevos procesos, patentes, modelos de gestión, la marca, las tecnologías y el aprovechamiento de las bases de datos que permiten mejorar los mecanismos de relación con los proveedores, clientes, entidades del estado, e incluso con la competencia.

Los valores organizacionales como el capital intelectual se tornan semejantes, tanto en la correlación causal como en la recopilación de la información, lo que indica que los valores organizacionales son el soporte para la organización (Ramírez et al., 
2005; Romero \& Izarra, 2014; Velásquez et al., 2012), son creados como parte de su estructura y procesos (capital estructural), practicados por el capital humano y divulgados por capital relacional de la entidad financiera (Ramírez et al., 2005). Una inversión mayor en el capital intelectual mejora la eficiencia, la creación de valor e influye en el rendimiento de las entidades bancarias (Vidyarthi, 2019; Wang et al., 2018).

Desde una perspectiva más amplia, los valores organizacionales están en todos los procesos que realicen dentro y fuera de la organización, ya sea al momento de monitorear un sistema, planificar un proyecto, trabajar en equipo, relacionarse con las personas dentro y fuera de la entidad, al momento de establecer un contacto con una organización o proveedor, siempre será necesario tener los valores de la organización en mente. En definitiva, los valores organizacionales necesitan del capital humano para ser compartidos, divulgados, interiorizados, en todos los procesos involucrando al capital estructural y capital relacional que permiten alcanzar los objetivos y metas planteadas, determinándole a la entidad alcanzar el éxito empresarial.

\section{Referencias}

Alaminos, A., Francés, F. J., Penalva, C., \& Santacreu, Ó. A. (2015). Introducción a los Modelos Estructurales en Investigación Social (1st ed.). PYDLOS ediciones.

Alcover, C., Martínez, D., Rodríguez, F. \& Domínguez, R. (2004). Introducción a la psicología del trabajo. McGraw-Hill.

Araújo, A. (2015). La desigualdad salarial de género medida por regresión cuantílica: el impacto del capital humano, cultural y social. Revista Mexicana de Ciencias Políticas y Sociales, 60(223), 287-315. https://doi.org/10.1016/S0185-1918(15)72139-2

Arciniega, L., Woehr, D., \& Poling, T. (2008). El Impacto de la Diversidad de los Valores en los Equipos Sobre las Variables de Proceso y el Desempeño de la Tarea. Revista Latinuamericana de Psicología, 40(3), 523-538. http://www.redalyc.org/pdf/805/80511493009.pdf

Bollen, K. A. (1989). Structural Equations with Latent Variables. Wiley. https://doi.org/10.2307/2289630

Brooking, A. (1996). Intellectual capital. Core asset for the third millennium Enterprise (1st ed.). International Thomson Business Press.

Brown, T. A. (2015). Confirmatory Factor Analysis for Applied Research (2nd ed.). The Guilford Press. Browne, M. W., \& Cudeck, R. (1992). Alternative Ways of Assessing Model Fit. Sociological Methods \& Research, 21(2), 230-258. https://doi.org/10.1177/0049124192021002005

Byrne, B. M. (2010). Structural Equation Modeling with AMOS: Basic Concepts, Applications, and Programming (2nd ed.). Routledge. https://doi.org/10.1080/10705511.2014.935842

Camps, V. (2015). Los valores éticos de la profesión sanitaria. Educación Médica, 16(1), 3-8. https://doi.org/10.1016/j.edumed.2015.04.001

Catena, A., Ramos, M. M., \& Trujillo, H. M. (2003). Análisis multivariado. Un manual para investigadores. Biblioteca Nueva. Recuperado de: https://bit.ly/2PZZ67y

Chahal, H., \& Bakshi, P. (2016). Measurement of Intellectual Capital in the Indian Banking Sector. Vikalpa: The Journal for Decision Makers, 41(1), 61-73. https://doi.org/10.1177/025 6090916629253

Chiavenato, I. (2009). Gestión del talento humano (4th ed.). The McGrau-Hill Companities.

Demo, G., Fernandes, T., \& Fogaça, N. (2017). A influência dos valores organizacionais na percepção de políticas e práticas de gestão de pessoas. REAd. Revista Eletrônica de Administração (Porto Alegre), 23(1), 89-117. https://doi.org/10.1590/1413-2311.093.57040

Demuner, M. del R., Saavedra, M. L., \& Camarena, M. E. (2017). Medición del capital intelectual en el sector bancario: Aplicación de los modelos Skandia y VAIC. Innovar, 27(66), 75-89. https://doi.org/10.15446/innovar.v27n66.66712 
Edvinsson, L., \& Malone, M. (2004). Capital Intelectual: Cómo identificar y calcular el valor inexplorado de los recursos intangibles de su empresa (19th ed.). Editorial Norma.

Edvinsson, L., \& Sullivan, P. (1996). Developing a model for managing intellectual capital. European Management Journal, 14(4), 356-364. https://doi.org/10.1016/0263-2373(96)00022-9

Estivalete, V. de F. B., \& Andrade, T. de. (2012). A influência dos valores organizacionais na percepção de suporte organizacional com base na concepção dos colaboradores do setor bancário. RAM. Revista de Administração Mackenzie, 13(3), 214-244. https://doi. org/10.1590/S1678-69712012000300010

Euroforum. (1998). Medición del capital intelectual: modelo Intelect. Euroforum Escorial.

Eyzaguirre, L. de F. (2017). Influencia del capital intelectual en la mejora de la producción científica de la universidad pública peruana [Universidad Nacional Mayor de San Marcos]. http://bit.ly/2ZtjjEz

Fernández-Jardón, C. M., \& Martos, M. S. (2016). Capital intelectual y ventajas competitivas en pymes basadas en recursos naturales de Latinoamérica. Revista Innovar Journal, 26(60), 117-132. https://doi.org/10.15446/innovar.v26n60.55548

Fernández, E., Montes, J., \& Vázquez, C. (2010). Los recursos intangibles como factores de competitividad de la empresa. Departamento de Administración de Empresas y Contabilidad, 1(1), 20-98. Recuperado de: https://bit.ly/2U8wY4W

Fitzgerald, G. A., \& Desjardins, N. M. (2004). Organizational Values and Their Relation to Organizational Performance Outcomes. Atlantic Journal of Communication, 12(3), 121-145. https://doi.org/10.1207/s15456889ajc1203_1

Ghozali, I. (2006). Structural equation modeling. Metode alternative dengan partial least squares. Diponegoro University Publishing Agency.

Hair, J. F., Anderson, R. E., Tatham, R. L., \& Black, W. C. (2007). Análisis multivariante (A. Otero (ed.); 5th ed.). Pearson Prentice Hall.

Hassan, A. (2007). Human resource development and organizational values. Journal of European Industrial Training, 31(6), 435-448. https://doi.org/10.1108/03090590710772631

Hayes, A. F. (2013). Introduction to Mediation, Moderation and Conditional Process Analysis: A Regression-Based Approach (9th ed.). The Guilford Press. https://doi.org/10.1017/CBO9781107 415324.004

Hernández, J. A., Espinoza, J. de J., \& Aguilar, M. (2015). Diferencias en los motivadores y los valores en el trabajo de empleados en empresas maquiladoras. Contaduría y Administración, 1-26. https://doi.org/10.1016/j.cya.2015.09.003

Herrera, E. E., \& Macagnan, C. B. (2015). Revelación de informaciones sobre capital estructural organizativo de los bancos en Brasil y España. Contaduría y Administración, 1-22. https://doi.org/10.1016/j.cya.2015.09.007

Ibarra-Cisneros, M. A., \& Hernández-Perlines, F. (2019). La influencia del capital intelectual en el desempeño de las pequeñas y medianas empresas manufactureras de México: el caso de Baja California. Innovar, 29(71), 79-96. https://doi.org/10.15446/innovar.v29n71.76397

Jaakson, K. (2010). Management by values: Are some values better than others? Journal of Management Development, 29(9), 795-806. https://doi.org/10.1108/02621711011072504

Javier, D. R., \& Quintana, A. M. C. (2017). Competencias y valores organizacionales de consejeros y corredores de seguros en la ciudad de Arequipa en el año 2016. Universidad Nacional de San Agustín de Arequipa. Recuperado de: http://bit.ly/competenciasyvalores

Kido, A., \& Kido, M. T. (2015). Modelos teóricos del capital humano y señalización: un estudio para México. Contaduría y Administración, 60(4), 723-734. https://doi.org/10.1016/j. cya.2014.06.001

Kogut, B., \& Zander, U. (1996). What firms do? Coordination, identity, and learning. Organization Science, 7(5), 502-518.

León, A., \& Mancheno, M. (2017). Componentes del capital intelectual un enfoque hacia la innovación de las organizaciones. Revista Publicando, 12(2), 302-314. 
Manzano, A., \& Zamora, S. (2009). Sistema de ecuaciones estructurales: una herramienta de investigación. Cuaderno técnico 4 (Cuaderno t). Centro Nacional de Evaluación para la Educación Superior, A.C. (Ceneval).

Marchante, A. J., \& Ortega, B. (2010). Capital humano, desajuste educativo y productividad del trabajo: Un estudio para la industria hotelera. Cuadernos de Economía y Dirección de La Empresa, 13(44), 79-100. https://doi.org/10.1016/S1138-5758(10)70020-7

Martín, G., Alama, E. M., Navas, J. E., \& López, P. (2009). El papel del capital intelectual en la innovación tecnológica. Un aplicación a las empresas de servicios profesionales de España. Cuadernos de Economía y Dirección de La Empresa, 12(40), 83-109. https://doi. org/10.1016/S1138-5758(09)70043-X

Martínez, I. M., \& Cegarra, J. G. (2003). Gestion dinámica del capital intelectual desde la perspectiva de los indicadores externos. Congreso Nacional de la Asociación Científica de Economía y Dirección de la Empresa, 1-21. Recuperado de: https://bit.ly/2vpD1rs

Mercado-Salgado, P. (2016). Validez Inicial de una Escala de Medición del Capital Intelectual en Universidades. Universitas Psychologica, 15(2), 109-120. https://doi.org/10.11144/Javeriana.upsy15-2.viem

Monagas, M. (2012). El capital intelectual y la gestión del conocimiento. Ingeniería Industrial, 33(2), 142-150. Recuperado de: http://bit.ly/2X6o7i7

Morales, L. E. (2017). Contribución del capital intelectual como fuente de ventaja competitiva de las organizaciones. Revista Multi-Ensayos, 3(5), 29-37. Recuperado de: https://bit.ly/2uDP5Ff

Navas, J. E., \& Ortiz, M. (2002). El capital intelectual en la empresa. análisis de criterios y clasificación multidimencional. Economia Industrial, 346(4), 163-171. Recuperado de: https:// bit.ly/36Flicy

Oliveira, A., \& Tamayo, A. (2004). Inventário de perfis de valores organizacionais. Revista de Administração, 39(2), 129-140.

Oro, I. M., Tonidandel, É. D., Müller, C. M., \& Sott, V. R. (2017). Divulgação do capital intelectual e a relação com o desempenho das melhores empresas para você trabalhar. Revista de Finanças e Contabilidade Da Unimep - REFICONT, 4(1), 38-54. Recuperado de: https:// bit.ly/2O8K0eO

Ortiz, F. A. A., \& Arenas, A. J. (2015). La gestión del conocimiento un desafío para las instituciones educativas en Colombia: emergencias y tensiones desde la teoría del capital intelectual. Gestión de la educación, 5(2), 137-150. https://bit.ly/2Q1eMYb

Osorio, M. (2003). El capital intelectual en la gestión del conocimiento. ACIMED, 11(6). Recuperado de: http://bit.ly/2X393BL

Pérez, Y., \& Coutín, A. (2005). La gestión del conocimiento: un nuevo enfoque en la gestión empresarial. Acimed, 13(6). Recuperado de: http://bit.ly/2J7vili

Pizarro, I., Real, J. C., \& Dolores de la Rosa, M. (2011). La incidencia del capital humano y la cultura emprendedora en la innovación. Cuadernos de Economía y Dirección de La Empresa, 14(3), 139-150. https://doi.org/10.1016/j.cede.2010.09.001

Ramírez, F., Sánchez, M., \& Quintero, H. (2005). El papel de los valores en el desarrollo de la identidad corporativa. Revista Negotium, 1(1), 35-54. Recuperado de: https://bit.ly/2GAHev0

Raykov, T., \& Marcoulides, G. A. (2006). A First Course in Structural Equation Modeling (2nd ed.). Lawrence Erlbaum Associates.

Revilla, E. (2013). Los valores organizacionales. el caso de un instituto pedagógico público de Lima. Pontificia Universidad Católica del Perú. Recuperado de: https://bit.ly/2GAp9gm

Rimbau-Gilabert, E., \& Myrthianos, V. (2014). Contratación de la mano de obra y desempeño en los servicios intensivos en conocimiento: una visión del capital intelectual. Intangible Capital, 10(2), 376-399. Recuperado de: https://bit.ly/36HFuL7

Romero, A. T., \& Izarra, K. (2014). Valores organizacionales de la responsabilidad social empresarial en las empresas del sector inmobiliario en el Municipio Maracaibo. Revista Electrónica de Gerencia Empresarial, 6(1), 30-41. Recuperado de: https://bit.ly/2vx2QGh 
Saboya, N., Loaiza, O. L., Soria, J. J., \& Bustamante, J. (2019). Fuzzy Logic Model for the Selection of Applicants to University Study Programs According to Enrollment Profile. In Advances in Intelligent Systems and Computing (Vol. 850, pp. 121-133). Springer Verlag. https://doi.org/10.1007/978-3-030-02351-5_16

Sánchez, A. J., Melián, A., \& Hormiga, E. (2007). El concepto de capital intelectual y sus dimensiones. Investigaciones Europeas de Dirección y Economía de La Empresa, 13(2), 97-111. https://doi.org/ISSN:1135-2523

Sánchez, M. (2012). El capital intelectual y su relación con diferentes gestiones: estudio teórico-conceptual. Ciencias de la Información, 43(3), 3-13. Recuperado de: https://bit.ly/2Gz3qp7

Santos, H., Figueroa, P., \& Fernández, C. (2011). El capital estructural y la capacidad innovadora de la empresa. Investigaciones Europeas de Dirección y Economía de La Empresa, 17(3), 69-89. https://doi.org/10.1016/S1135-2523(12)60121-X

Sarur, M. S. (2013). La importancia del capital intelectual en las Organizaciones. Ciencia Administrativa, 1, 39-45. Recuperado de: https://bit.ly/2GwUzUO

Schein, E. H. (2004). Organizational Culture and Leadership (3rd ed.). Jossey-Bass. http://bit.ly/2J7lXty

Schumacker, R. E., \& Lomax, R. G. (2010). A Beginner's Guide to Structural Equation Modeling (3rd ed.). Routledge.

Schwartz, S. H. (1992). Universals in the content and structure of values: Theoretical advances and empirical tests in 20 countries. Advances in Experimental Social Psychology, 25, 1-65. https://doi.org/10.1016/S0065-2601(08)60281-6

Sen, A. (1998). Human capital and human capacity. Cuadernos de economía (Santafé de Bogotá), 17(29), 67-72. Recuperado de: http://bit.ly/2J6POlX

Siles, M. M. (2013). Competencias profesionales requeridas por las empresas en Tarapoto. Apuntes Universitarios, 3(2), 19-38. Recuperado de: http://bit.ly/2J7utsI

Tabachnick, B. G., Fidell, L. S., \& Ullman, J. B. (2007). Using Multivariate Statistics (Allyn \& Bacon (ed.)). Pearson Education.

Tamayo, A. (2007). Impacto dos Valores da Organização sobre o Estresse Ocupacional. RAC Eletronica, 1(2), 20-33. Recuperado de: http://bit.ly/2ZUqIh1

Valencia, M. (2005). El capital humano, otro activo de su empresa. Entramado, 1(2), 20-33. https://doi.org/DOI: 10.1590/1413-82712016210105

Velásquez, Y. (2007). Modelos de Productividad basado en Valores Organizacionales. XI Congreso de Ingeniería de Organización, 677-686. Recuperado de: https://bit.ly/3aRBXgn

Velásquez, Y. (2015). Modelo explicativo de la relación entre productividad y valores organizacionales, en la PYME del sector metalúrgico y minero de Venezuela. E.T.S.I. Industriales (UPM). Recuperado de: http://bit.ly/2FCjshQ

Velásquez, Y., Rodríguez, C., \& Guaita, W. (2012). Los valores Organizacionales: Referencia para la evaluación de la productividad. 6th International Conference on Industrial Engineering and Industrial Management., 6th, 840-846. Recuperado de: http://bit.ly/2LlIkOM

Vidal, C. R. (2017). Modelo de capital intelectual para la investigación en las universidades públicas de la Costa Caribe colombiana. Actualidades Investigativas En Educación, 17(1), 1-27. https://doi.org/10.15517/aie.v17i1.27332

Vidyarthi, H. (2019). Dynamics of intellectual capitals and bank efficiency in India. Service Industries Journal, 39(1), 1-24. https://doi.org/10.1080/02642069.2018.1435641

Villalobos, J. (2014). Valores organizacionales en la filosofía de gestión para instituciones educativas Bolivarianas. REDHECS: Revista electrónica de Humanidades, Educación y Comunicación Social, 9(16), 35-48. Recuperado de: http://bit.ly/2xda3sJ

Villegas, E., Hernández, M. A., \& Salazar, B. C. (2017). La medición del capital intelectual y su impacto en el rendimiento financiero en empresas del sector industrial en México. Contaduría y Administración, 62(1), 184-206. https://doi.org/10.1016/j.cya.2016.10.002

Wang, Z., Cai, S., Liang, H., Wang, N., \& Xiang, E. (2018). Intellectual capital and firm performance: the mediating role of innovation speed and quality. International Journal of $\mathrm{Hu}$ - 
man Resource Management, O(0), 1-29. https://doi.org/10.1080/09585192.2018.1511611

Westland, J. C. (2019). Structural Equation Models: From Paths to Networks (2nd ed.). Springer. https://doi.org/10.1007/978-3-030-12508-0

Williams, S. L. (2002). Strategic planning and organizational values: Links to alignment. Human Resource Development International, 5(2), 217-233. https://doi. org $/ 10.1080 / 13678860110057638$

Yangali, J. S., \& Quiróz, V. S. (2018). Valuación del capital intelectual, su contabilización y presentación como activo intangible en los estados financieros. INNOVA Research Journal, 3(11), 35-61. https://doi.org/10.33890/innova.v3.n11.2018.889

Yarce, J. (2000). Los valores son una ventaja competitiva. (Ágora Editores Ltda, Ed.). Instituto Latinuamericano de Liderazgo (ILL). Recuperado de: https://bit.ly/2tYN8De 